\title{
Estimación de una función para la producción de compost: el caso europeo
}

\author{
Estimação de uma função para a produção de composto: o caso europeu \\ Estimation of a function for the production of compost: the european case \\ David Andrés Camargo Mayorga ${ }^{1}$ (D), Octavio Cardona García ${ }^{1}{ }^{(\mathbb{D})}$, Juan Manuel González Guzmán \\ ${ }^{1}$ Universidad Militar Nueva Granada, Bogotá, Colombia. E-mails: david.camargo@unimilitar.edu.co; \\ octavio.cardona.g@gmail.com; juan.gonzalez@unimilitar.edu.co
}

\begin{abstract}
Cómo citar: Camargo Mayorga, D. A., Cardona García, O., \& González Guzmán, J. M. (2020). Estimación de una función para la producción de compost: el caso europeo. Revista de Economia e Sociologia Rural, 58(3), e192632. https://doi.org/10.1590/18069479.2020 .192632
\end{abstract}

Resumen: Este artículo tiene como objetivo estimar una función de producción de compost para 31 países de Europa usando la metodología de panel de datos. La motivación del documento se basó en el rezago que existe en la literatura económica, sobre temas relacionados con el tratamiento de residuos sólidos y su posible reintegración al mercado. Se encontró que la función de producción que mejor se ajusta es la translogarítmica. Al calcular las elasticidades de sustitución de Allen se halló que el capital, representado por el índice de instalación de nuevas plantas de tratamiento, es complementario con los demás factores de producción. Caso contrario ocurrió con el trabajo y los residuos municipales generados, siendo sustitutivos para el grupo de países analizados.

Palabras clave: función de producción, residuos sólidos, compost, Europa.

Resumo: Este artigo pretende estimar uma função de produção de composto para 31 países da Europa usando a metodologia do painel de dados. A motivação do documento fundamentou-se no atraso que existe na literatura econômica quanto a questões relacionadas ao tratamento de resíduos sólidos e sua possível reintegração no mercado. Verificou-se que a função de produção que melhor se adapta é translogarítmica. Ao calcular as elasticidades de substituição de Allen, descobriu-se que o capital, representado pela taxa de instalação de novas estações de tratamento, é complementar aos outros fatores de produção. Ocorreu o contrário com o trabalho e o desperdício municipal gerado, sendo substitutos do grupo de países analisados.

Palavras-chave: função de produção, resíduos sólidos, composto, Europa.

Abstract: This paper aims to estimate a compost production function for 31 countries in Europe using the data panel methodology. The motivation of the document was based on the lag that exists in the economic literature, on issues related to the treatment of solid waste and its possible reintegration into the market. It was found that the production function that best fits is translogarithmic. When calculating Allen's elasticities of substitution, it was found that capital is complementary to the other factors of production, represented by the rate of installation of new treatment plants,. The opposite occurred with the work and the municipal waste generated, being substitutes for the group of countries analyzed.

Keywords: production function, solid waste, compost, Europe. 


\section{Introducción}

El presente documento tiene como objetivo elaborar una aproximación de la función de producción de compost para 31 países de Europa. La motivación del documento radica principalmente en el marcado rezago que existe en la literatura económica, sobre temas relacionados con el tratamiento de residuos sólidos y su posible reintegración al mercado.

Algunos de los antecedentes que se encuentran en la literatura consultada, indican que efectivamente ha habido algunos estudios dedicados al mismo tema, y aunque no todos se manejan dentro de la misma metodología o son aplicaciones específicas o estudios de caso, su contribución es considerable teniendo en cuenta la escases de los mismos.

Dentro de los estudios encontrados se identifica que los análisis están restringidos a los rendimientos sobre la producción de determinados tipos de cultivos, que tiene la utilización del compost en suelo. Rara vez se rastrean documentos que se dediquen a analizar la producción de este insumo per se, pero dentro de los documentos que se pudieron encontrar, también hay una manifestación explicita de la dificultad para relacionar precios o identificar la forma del mercado de compostaje.

En el tema relativo a la metodología y el modelo utilizado, las características de los datos permiten ejecutar un análisis de datos panel con una función de producción translogarítmica. Dado que existen algunos valores perdidos, el modelo es desbalanceado. La función está especificada de forma tal que se considere a la cantidad en toneladas de compost en función de las plantas instaladas, la cantidad de residuos municipales generada, y el empleo agrícola como porcentaje del empleo total.

La organización del documento viene dada primero por la presente introducción; en segundo lugar, antecedentes; tercero, metodología; cuarto, resultados, quinto, discusión de resultados y sexto, conclusiones.

\section{Antecedentes}

El compostaje, al igual que otros temas relacionados con el medio ambiente, es una solución para la disposición de residuos sólidos bastante plausible y fácil de aplicar a una pequeña escala. No obstante, la literatura consultada hasta el momento en que se escribió este documento rara vez expone un estudio analizando el tema desde una perspectiva económica, y cuando este es el caso, existen algunos limitantes en su exposición, o mantienen la misma línea argumental inclinada hacía análisis técnicos y bioquímicos. (Camargo et al., 2017b).

Algunas causas de esta falta de estudios se pueden explicar por la falta de un sistema de precios pues el precio marginal es cercano a cero (Park et al., 2002). Por otro lado, pueden existir algunos factores no considerados por la literatura: la falta de una teoría definida, confusión en algunos términos económicos, falta de incentivos para realizar estudios relacionados con el tema, elevados costos de capital, costos marginales cercanos a cero, entre otros, son mencionados con más detalle en Camargo et al. (2017a).

Ahora bien, aunque no son muchos, existen algunos estudios en los que se trabaja directamente ya sea de forma cualitativa o cuantitativa el tema del compostaje. En la mayoría de los casos, se estudia al compost desde su participación como fertilizante y se trata de determinar su efectividad sobre el suelo, es decir su impacto sobre la productividad de determinado cultivo.

En este orden de ideas, se puede encontrar trabajos como el de Arancon et al. (2004), en donde se analiza el efecto del compostaje sobre el rendimiento de los suelos en california y como el de Jackson et al. (2004), el cuál expone análisis quimicos, pero también estadísticos y económicos, relacionados directamente con los cambios en productividad que el uso de compostaje tiene sobre diferentes tipos de cultivo de fresa.

Por otra parte, en lo relacionado con el uso de funciones Cobb-Douglas, los estudios consultados mantienen la misma línea, es decir, utilizan el compost como una variable explicativa de la producción agrícola, algunos de estos estudios se concentran en análisis de 
datos de determinadas áreas rurales o incluso espacios privados como haciendas y tierras de cultivo. Dichos estudios se resumen en la siguiente Tabla 1:

Tabla 1 Estudios cuantitativos para funciones de producción de compost usando la forma Cobb-Douglas

\begin{tabular}{|c|c|}
\hline Autor & Resumen \\
\hline $\begin{array}{c}\text { Grabowski } \\
\text { (1985) }\end{array}$ & $\begin{array}{l}\text { En este artículo tratan de medir la eficiencia técnica de } 537 \text { granjas en Nepal. La metodología } \\
\text { de estimación de la función es mediante mínimos cuadrados ordinarios. Algunos resultados } \\
\text { que destacan los autores son las diferenciaciones en términos de eficiencia (mejorías en la } \\
\text { producción de los cultivos) entre diferentes cultivos como el arroz y el maíz cuando se abonan } \\
\text { con el producto del compost. }\end{array}$ \\
\hline $\begin{array}{c}\text { Folefack } \\
(2009)\end{array}$ & $\begin{array}{l}\text { El autor de este documento analiza la posibilidad de sustitución de fertilizantes químicos por } \\
\text { compost. Un aspecto que llama bastante la atención de este trabajo es que, para el caso } \\
\text { camerunés, los precios del mercado de compost, están por encima de los costos de } \\
\text { producción, por lo tanto hay posibilidades de la existencia de un mercado. Dentro de los } \\
\text { resultados destacables, se halla que efectivamente puede existir una relación de sustitución, } \\
\text { pero es necesario impulsar más este tipo de políticas en el país africano. }\end{array}$ \\
\hline $\begin{array}{c}\text { Folefack \& } \\
\text { Adamowski } \\
\text { (2012) }\end{array}$ & $\begin{array}{l}\text { El objetivo de este documento es desarrollar una metodología que permita estimar la } \\
\text { rentabilidad de la producción de compost sujeta al criterio de transporte. El enfoque del } \\
\text { artículo es bastante interesante en el sentido de que agrega el espacio como un factor muy } \\
\text { importante dentro de la producción de compost a las afueras de un poblado en Camerún. La } \\
\text { metodología utilizada en este documento es la aplicación del modelo von Thünen, } \\
\text { relacionando la distancia de distribución y consumo con el producto marginal de la tierra. } \\
\text { Dentro de los resultados más destacables se encuentra que la producción de este insumo es } \\
\text { rentable siempre y cuando pueda ser transportada a no más de } 79 \text { kilómetros a la redonda de } \\
\text { la ciudad de estudio, lo que significa que cualquier granja salida de dicho rango estaría por } \\
\text { fuera de las denominadas "zonas de cultivo beneficiosas". }\end{array}$ \\
\hline
\end{tabular}

Fuente: Elaboración propia

En otro orden de ideas, el tratamiento de residuos sólidos ha venido fortaleciéndose más en países de ingresos altos, aquellos que pueden pagar las plantas de tratamiento y su mantenimiento, esto explica la participación única de países europeos en el modelo que se muestra páginas más adelante.

Sumado a lo anterior, el hecho de que los países objeto de este estudio sean todos europeos, influye en la forma como se integra el trabajo a la producción de compostaje. En Europa la organización legal prohíbe la inadecuada disposición de residuos desde hace treinta años aproximadamente, dejando como única opción la quema de los mismos o su reintegro al sistema productivo.

En la Figura 1, se explica el marco en el que se desenvuelve Europa actualmente, y su influencia sobre la producción y uso de compostaje en las actividades productivas de la región.

\begin{tabular}{|l|}
\hline \multicolumn{1}{|c|}{ Marco legal } \\
\hline $\begin{array}{l}\text {-Directiva 2000/60/EC sobre el cuidado } \\
\text { del agua } \\
\text {-Directiva } 91 / 271 / \mathrm{EC} \text { sobre el } \\
\text { tratamiento urbano de aguas }\end{array}$ \\
-Directiva 99/31/EC sobre el vertedero \\
de residuos \\
-Directiva 86/278/EEC sobre el uso de \\
lodos en la agricultura
\end{tabular}

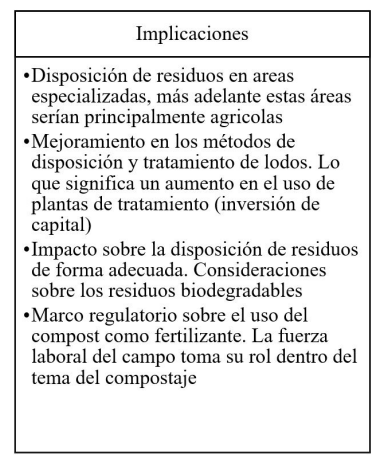

Figura 1 Marco legal sobre tratamiento de agua y residuos en la Unión Europea.

Fuente: Elaboración propia 
El marco legal diseñado en la zona euro favorece bastante el uso de los residuos sólidos, en comparación con otras regiones. Aunque dentro de la revisión se encuentran estudios pertenecientes a otros lugares como África y Asia, la disponibilidad de datos y recursos se concentra principalmente en Europa.

Este marco legal tiene en cuenta que los residuos destinados específicamente al compostaje necesitan grandes áreas de tierra, por eso la "European Compost Network" que es una entidad promotora del método de compostaje en la Unión Europea, ha reforzado campañas para la promoción del uso de compost como abono para la agricultura ecológica. Lo mencionado implica que la mano de obra en la agricultura tiene efectos sobre el compostaje en dicha región y no es un fenómeno completamente urbano.

\section{Metodología}

Para realizar el presente trabajo se utiliza como muestra una serie de datos desde el 2004 hasta el 2016 para 31 países $^{1}$ ubicados en Europa. Las variables consideradas para el modelo son: producción de compost en miles de toneladas como variable dependiente; generación de residuos municipales en miles de toneladas; índice de plantas de tratamiento de aguas residuales instaladas o removidas por año, con año base 2016=100²; por último, el nivel de empleo en agricultura como porcentaje del total de empleo de cada país. Estas últimas tres son las variables independientes del modelo. La fuente de esta serie es Passport-Euromonitor para el caso de la producción de compost, los residuos municipales y las plantas de tratamiento, y el Banco Mundial para el nivel de empleo en agricultura.

Algunas estadísticas descriptivas de todas las variables se presentan en la siguiente Tabla 2:

Tabla 2 Estadísticos descriptivos de las variables

\begin{tabular}{cccccc} 
Variable & Observaciones & Media & Desv. Estándar & Mínimo & Máximo \\
Y & 371 & 1235.20 & 2044.90 & 1.00 & 9494.00 \\
M & 403 & 9146.32 & 12608.10 & 97.00 & 50237.00 \\
K & 403 & 94.96 & 18.53 & 33.33 & 175.61 \\
L & 403 & 6.70 & 6.49 & 0.95 & 33.96 \\
\hline
\end{tabular}

Fuente: Elaboración propia

En la tabla se puede identificar que la variable dependiente (representada por Y), tiene algunos valores perdidos, estas observaciones faltantes, no se encuentran en todos los países, simplemente durante algunos años en determinados países no hay una medida de la producción de compost. Por lo tanto, estamos tratando un panel de datos desbalanceado.

De otra manera, el valor medio de la producción de compost durante todo el periodo especificado, acorde a los datos existentes, fue de aproximadamente 1,235,000 toneladas, con un valor mínimo de 1,000 toneladas y un máximo de alrededor de 9 millones.

Análogamente, los residuos municipales (M) producidos en las mismas fechas, totalizaron más de nueve millones de toneladas en promedio, con un valor mínimo de 97 mil y un máximo de más de 50 millones de toneladas.

\footnotetext{
${ }^{1}$ Los países con disponibilidad de datos fueron: Austria, Bélgica, Bulgaria, Croacia, Chipre, República Checa, Dinamarca, Estonia, Finlandia, Francia, Alemania, Grecia, Hungría, Islandia, Irlanda, Italia, Letonia, Lituania, Luxemburgo, Malta, Holanda, Noruega, Polonia, Portugal, Rumania, Eslovaquia, Eslovenia, España, Suecia, Turquía y Reino Unido.

${ }^{2}$ Esta variable se construye teniendo en cuenta que el número de plantas de tratamiento es una variable agregada. Para su construcción se toma el número de plantas existentes cada año y se divide por las plantas existentes en el año 2016.
} 
Con respecto al índice de plantas de tratamiento instaladas $(\mathrm{Y})$, en comparación a la cantidad de cada país para el año 2016, en promedio han existido el $94 \%$ de dicho valor durante todo el periodo especificado para cada país. En otro sentido, el valor máximo del índice de plantas instaladas (175.6\%) sugiere que, para algún país de la muestra, en algún momento del tiempo, su número de plantas de tratamiento casi duplicaba la cantidad que había en el 2016. Adicionalmente, porcentaje de empleo en agricultura, sobre el total de empleo del país (L), promedió 6.7\% con un máximo de 33.96\% y un mínimo de $0.95 \%$.

En otro orden de ideas, dos limitaciones que pueden mencionarse de los datos incluyen el hecho de la escases de los mismos, sería ideal disponer de la producción de compost de cada país del mundo así sea en cortos periodos de tiempo. En segundo lugar, para el tema del capital invertido y el trabajo, las variables que se utilizan en el presente trabajo son meras aproximaciones, no hay una base de datos que concentre la información necesaria acerca de inversiones para el tratamiento de residuos y mucho menos para el compostaje, así mismo el número de empleos que este rubro genera es difuso.

\subsection{Especificación econométrica}

La metodología planteada desde el principio es un panel de datos desbalanceado. No obstante, la especificación matemática de la función debe ser determinada econométricamente. En primera instancia se parte de una función clásica de tipo Cobb-Douglas, que toma la siguiente forma:

$Y=A K^{\alpha} L^{\beta} M^{\delta}$

La mencionada función en el caso de la presente metodología viene representada en cada una de sus variables, como sigue:

$Y=$ Producción de compost

$K=$ índice de plantas de tratamiento instaladas, como variable proxy del capital

$L=$ Porcentaje del trabajo agrícola como variable proxy del trabajo

$M=$ Generación de residuos municipales

$A=$ Es la constante que en la teoría tradicional se interpreta como el nivel tecnológico o productividad de los factores

En términos econométricos la función Cobb-Douglas debe ser linealizada, es decir que deben aplicarse logaritmos naturales para poder estimar los coeficientes por diferentes metodologías esencialmente lineales como es el caso de los datos panel.

Aunque la función de producción de la forma anteriormente expuesta es una de las más populares, en la literatura han venido apareciendo diferentes especificaciones un poco más flexibles, que prometen obtener resultados más acordes a la realidad y la estructura de los datos.

Una de estas especificaciones es la llamada función translogarítmica. Esta forma funcional fue usada por primer vez en el trabajo de Christensen et al. (1973) y para los propósitos de este trabajo, tiene la siguiente forma ${ }^{3}$ :

$$
\begin{aligned}
& \operatorname{LnY}=\mu_{0}+\mu_{1} \operatorname{Ln} K_{i t}+\mu_{2} \operatorname{Ln} L_{i t}+\mu_{3} \operatorname{LnM}_{i t}+\mu_{4}\left(1 / 2 \operatorname{Ln}^{2} K_{i t}\right)+\mu_{5}\left(1 / 2 \operatorname{Ln}^{2} L_{i t}\right)+ \\
& \mu_{6}\left(1 / 2 \operatorname{Ln}^{2} M_{i t}\right)+\mu_{7} \operatorname{Ln} K_{i t} \operatorname{Ln} L_{i t}+\mu_{8} \operatorname{Ln} K_{i t} \operatorname{Ln} M_{i t}+\mu_{9} \operatorname{Ln} L_{i t} \operatorname{Ln} M_{i t}+\varepsilon_{i t}
\end{aligned}
$$

\footnotetext{
${ }^{3}$ La función dada se presenta sin efectos, para conocer las especificaciones de las funciones con efectos fijos y aleatorios, y otras especificaciones y detalles acerca de los modelos, el lector puede consultar el trabajo de Ramíres (2015) y el libro de Greene (1998).
} 
En el modelo 2, el subíndice $i$ represta el i-esimo país de la serie y $t$ es el t-esimo periodo.

A diferencia del modelo Cobb-Douglas, donde las elasticidades calculadas como la primera derivada de la función, son constantes, en el modelo translogarítmico las elasticidades de cada factor de producción son variables en cada sujeto y momento del tiempo. El cálculo de estas elasticidades se especifica en las Ecuaciones 3, 4 y 5.

$$
\begin{aligned}
& \varepsilon_{k}=\mu_{1}+\mu_{4} L n K_{i t}+\mu_{7} L n L_{i t}+\mu_{8} L n M_{i t} \\
& \varepsilon_{l}=\mu_{2}+\mu_{5} \operatorname{Ln} L_{i t}+\mu_{7} \operatorname{Ln} K_{i t}+\mu_{9} L n M_{i t} \\
& \varepsilon_{m}=\mu_{3}+\mu_{6} L n M_{i t}+\mu_{8} \operatorname{Ln} K_{i t}+\mu_{9} L n L_{i t}
\end{aligned}
$$

Con base en la misma forma de análisis, para las funciones translogarítmicas se puede aplicar el concepto de elasticidad de sustitución parcial de Allen (1938), que puede ser calculada como sugiere Greene (1998) de la siguiente manera:

$$
\theta_{i j}=\frac{\mu_{i j}+\varepsilon_{i} \varepsilon_{j}}{\varepsilon_{i} \varepsilon_{j}} ; \theta_{i j}=\frac{\mu_{i i}+\varepsilon_{i}\left(\varepsilon_{i}-1\right)}{\varepsilon_{i}^{2}}
$$

En donde $\mu_{i j}$ es el coeficiente del producto de cada variable y $\varepsilon$ la elasticidad de cada variable.

\section{Resultados}

Antes de presentar los resultados es necesario mostrar las pruebas previas que permiten identificar el tipo de modelo que debe ser aplicado. En la Tabla 3 se comparan las diferentes pruebas realizadas para conocer si el panel se comporta como un pool de datos, efectos fijos o efectos aleatorios ${ }^{4}$.

Tabla 3 Contrastes de especificación entre efectos fijos y aleatorios

\begin{tabular}{cccc} 
Prueba & Estadístico & Valor crítico (5\%) & Modelo \\
F & 16.06 & 1.494 & Ha: Efectos Fijos \\
Breusch-Pagan & 341.13 & 16.91 & Ha: Efectos Fijos \\
Hausman & 52.85 & 3.84 & Ha: Efectos Fijos \\
\hline
\end{tabular}

Fuente: Elaboración propia

Una vez identificado el modelo, que en este caso es de efectos fijos, se procede a realizar las estimaciones y la prueba $\mathrm{F}$ para conocer si el modelo tiene la forma funcional restringida (Cobb-Douglas) o por el contrario es un modelo translogarítmico ${ }^{5}$. El valor calculado de la prueba fue 5.56 (algo que coincide ligeramente con la prueba de separabilidad total que se muestra más adelante) por lo tanto está por encima del valor crítico de 2.12, lo que significa que el modelo correcto es el translogarítmico.

\footnotetext{
${ }^{4}$ Los procedimientos completos se presentan en los Anexos A1, A2, A3, A4 y A5 de este documento.

5 Esta prueba se aplica sobre los coeficientes contrastando la hipótesis nula (Ho) de que $\mu_{4}=\mu_{5}=\mu_{6}=\mu_{7}=\mu_{8}=\mu_{9}=0$. Los resultados de esta prueba se presentan en el Anexo A6 de este documento.
} 
Para ilustrar los resultados, las salidas del modelo especificado en stata, se encuentran consignadas en la Tabla 4.

En este tipo de función el modelo tiene poco que pueda explicarse a simple vista en la tabla, sólo algunas anotaciones sobre la significancia global e individual de las variables, aquí se puede destacar que LnM no resulta significativa para el modelo en términos individuales, pero en término conjunto todas las variables si lo son.

Sumado a lo anterior, la "correlación intraclase" o rho, muestra que 94,9\% de la varianza es debido a diferencias a través del panel.

Tabla 4 Estimación del modelo por efectos fijos

$\begin{array}{cccc} & \text { Coeficiente } & \text { Errores estándar } & \text { P valor } \\ \text { LnK } & 15.32217 & 5.216093 & 0.004 \\ \text { LnL } & 5.915998 & 2.027554 & 0.004 \\ \text { LnM } & -1.130277 & 2.074111 & 0.586 \\ \text { Ln2K } & -2.316252 & 1.014559 & 0.023 \\ \text { Ln2L } & -1.324032 & 0.3174048 & 0.000 \\ \text { Ln2M } & 0.4172337 & 0.2479055 & 0.093 \\ \text { LnKLnL } & -0.7531397 & 0.3298623 & 0.023 \\ \text { LnKLnM } & -0.497218 & 0.1852794 & 0.008 \\ \text { LnLLnM } & -0.2074103 & 0.173841 & 0.234 \\ \text { Constante } & -26.10144 & 16.61957 & 0.117 \\ & \text { Variable dependiente: LnY } & \end{array}$

Fuente: Elaboración propia

Análogamente, el valor de la presente metodología se encuentra principalmente en la identificación de las elasticidades de los diferentes factores considerados. Basados en las ecuaciones representadas anteriormente en (3), (4) y (5), se identifica que las elasticidades del capital, el trabajo y los insumos, son respectivamente $-0,38,-1.23$ y -0.30 , para todo el conjunto de países estudiado. Se debe tener en cuenta que el cálculo se realiza sobre los valores medios de cada variable, puesto que este tipo de elasticidades como se mencionaba en el apartado anterior, no son constantes.

Los signos de estas elasticidades no son los esperados, pues teóricamente no es lógico obtener una elasticidad negativa de los factores de producción. A priori se podría considerar que los limitantes en los datos influyen sobre este resultado, no obstante, algunos autores como Felipe \& Adams (2005), Griliches \& Mairesse (1995), Klette \& Griliches (1996), Lucas (1970) y Romer (1987) mencionan que es bastante común encontrar este tipo de resultados (principalmente en la variable del capital) en los estudios empíricos que se realizan para diferentes sectores.

En el mismo orden de ideas, se intentó conocer la elasticidad por país que cada uno de los factores tiene, por lo tanto, en la Tabla 5 que se muestra adelante se resumen los resultados obtenidos. 
Tabla 5 Elasticidades de K, L y M por país

\begin{tabular}{|c|c|c|c|}
\hline & $\mathbf{K}$ & $\mathbf{L}$ & $\mathbf{M}$ \\
\hline Austria & -0.355 & -1.299 & -0.134 \\
\hline Bélgica & 0.791 & 0.377 & 0.204 \\
\hline Bulgaria & -0.405 & -1.723 & -0.388 \\
\hline Croacia & -0.404 & -2.270 & -0.747 \\
\hline Chipre & 0.160 & -0.800 & -1.139 \\
\hline República Checa & -0.521 & -0.877 & -0.365 \\
\hline Dinamarca & 0.123 & -0.466 & -0.128 \\
\hline Estonia & 0.754 & -0.685 & -1.144 \\
\hline Finlandia & -0.499 & -1.192 & -0.457 \\
\hline Francia & -1.105 & -1.119 & 0.767 \\
\hline Alemania & -0.436 & -0.290 & 1.117 \\
\hline Grecia & -1.468 & -2.652 & -0.353 \\
\hline Hungría & -0.445 & -1.278 & -0.215 \\
\hline Islandia & 1.014 & -0.731 & -1.744 \\
\hline Irlanda & -0.554 & -1.413 & -0.453 \\
\hline Italia & -1.505 & -1.472 & 0.627 \\
\hline Letonia & -0.225 & -1.820 & -1.159 \\
\hline Lituania & -0.699 & -2.119 & -0.891 \\
\hline Luxemburgo & 2.192 & 1.010 & -0.909 \\
\hline Malta & 1.413 & 0.684 & -1.241 \\
\hline Holanda & -0.748 & -0.725 & 0.173 \\
\hline Noruega & 0.105 & -0.403 & -0.387 \\
\hline Polonia & -1.981 & -2.949 & -0.045 \\
\hline Portugal & -0.268 & -2.063 & -0.143 \\
\hline Rumania & -2.550 & -3.949 & -0.417 \\
\hline Eslovaquia & -0.010 & -0.809 & -0.584 \\
\hline Eslovenia & -0.403 & -1.846 & -1.096 \\
\hline España & -1.425 & -1.591 & 0.470 \\
\hline Suecia & -0.056 & -0.278 & -0.067 \\
\hline Turquía & -1.806 & -3.546 & 0.441 \\
\hline ReinoUnido & -0.587 & 0.083 & 0.878 \\
\hline
\end{tabular}

Fuente: Elaboración propia

El resultado más notable de las elasticidades diferenciadas por país es que a nivel individual algunas veces sí se cumple con el signo esperado. Por ejemplo, para Bélgica, Chipre, Dinamarca, Estonia, Luxemburgo, Malta y Reino Unido, al menos el capital y el trabajo cumplieron con los signos esperados. 
Dentro de estas estimaciones destacan la de Bélgica y Luxemburgo, no sólo porque ambas cumplen con los signos esperados sino porque son resultados dispares. En Bélgica todas las elasticidades fueron inelásticas, con una sensibilidad mayor del capital. En Luxemburgo en cambio, las variaciones son muy elásticas en el capital, y en el trabajo son prácticamente unitarias.

Pasando a otro punto, ahora se pretende encontrar cuál es la elasticidad cruzada de estos factores para todos los países de la muestra. Los factores de producción se consideran complementarios cuando el signo de la elasticidad de sustitución es negativo y son sustitutivos cuando el signo es positivo (Berndt \& Christensen, 1973). Teniendo en cuenta lo anterior, los signos de cada una de las elasticidades de Allen estimadas sugieren que entre el capital y las demás variables existe una relación de complementariedad, mientras que el trabajo y los insumos, son sustitutivos.

Igualmente, para el caso de la elasticidad propia, es decir del factor con respecto a sí mismo, la interpretación que sugiere Terrones-Cordero \& Sánchez-Torres (2010), es que cuando el valor absoluto de dicha elasticidad es mayor a cero, el factor es elástico. Por lo tanto, acorde a las elasticidades en el sentido de Allen que se muestran en la Tabla 6, todos los factores son elásticos e influyen de una forma más que proporcional en el cambio de producción de compostaje.

Tabla 6 Elasticidades de sustitución parcial de Allen

\begin{tabular}{cccc} 
& $\mathbf{K}$ & $\mathbf{L}$ & $\mathbf{M}$ \\
$\mathrm{K}$ & -12.107 & $\cdot$ & $\cdot$ \\
$\mathrm{L}$ & -0.591 & 0.940 & $\cdot$ \\
$\mathrm{M}$ & -3.213 & 0.453 & 8.669 \\
\hline
\end{tabular}

Fuente: Elaboración propia

Complementando el punto anterior, es necesario realizar una última y tercera prueba que busca determinar la separabilidad de los factores. Este concepto en palabras de Ibarra Salazar \& García Pérez (2013, p. 157), “...representa la manera en que la tasa marginal de sustitución técnica entre dos factores responde a cambios en la cantidad de otro factor de producción".

Para ejemplificar esta afirmación en el caso de la producción de compost, si el aumento en el número de plantas de tratamiento hace que el producto marginal del empleo y los residuos municipales se trasladen en la misma proporción, se puede afirmar que los materiales y la mano de obra son separables del capital.

Con el propósito de conocer lo anterior, es necesario realizar unas pruebas de hipótesis sobre las variables estudiadas. La Tabla 7 resume cómo deben ser estas pruebas.

Tabla 7 Restricciones en los parámetros y separabilidad

\begin{tabular}{|c|c|c|c|}
\hline Tipo de separabilidad & $\begin{array}{c}\text { Relación entre } \\
\text { las } \\
\text { elasticidades } \\
\text { de sustitución }\end{array}$ & $\begin{array}{l}\text { Restricciones en los } \\
\text { parámetros de la función } \\
\text { translogarítmica }\left(H_{\circ}\right)\end{array}$ & Estadístico F \\
\hline Separabilidad completa & $\sigma_{k m}=\sigma_{k l}=\sigma_{l m}=1$ & $\mu_{k m}=0, \mu_{k l}=0, \mu_{l m}=0$ & 5.59* (Rechaza $\left.\mathrm{H}_{\circ}\right)$ \\
\hline Separabilidad LK-M & $\sigma_{k m}=\sigma_{k l}=1$ & $\mu_{l m}=0 ; \mu_{m m}=0$ & $2.02 * \star\left(\right.$ Aprueba $\left.\mathrm{H}_{\circ}\right)$ \\
\hline Separabilidad KM-L & $\sigma_{k l}=\sigma_{l m}=1$ & $\mu_{l m}=0 ; \mu_{l l}=0$ & $10.66^{* *}\left(\right.$ Rechaza $\left.\mathrm{H}_{\circ}\right)$ \\
\hline Separabilidad LM-K & $\sigma_{k l}=\sigma_{m k}=1$ & $\mu_{l l}+\mu_{l m}=0, \mu_{l m}+\mu_{m m}=0$ & $12.51 * *\left(\right.$ Rechaza $\left.\mathrm{H}_{\circ}\right)$ \\
\hline
\end{tabular}

Fuente: Adaptado de Ibarra Salazar \& García Pérez (2013) 
Apoyados en las pruebas anteriormente presentadas, existe evidencia de separabilidad entre el número de plantas instaladas y el trabajo agrícola, con respecto a la generación de residuos municipales. Lo anterior implica que un aumento en la generación de residuos sólidos hace que el producto marginal del empleo agrícola y de la instalación de nuevas plantas de tratamiento de aguas, se trasladen en la misma proporción, para la producción de compost.

\section{Discusión de resultados}

La primera consideración que se puede analizar en los resultados se relaciona directamente con la elasticidad simple de los factores en sus valores medios. Aunque los signos esperados se alejan de sus resultados teóricos, algunas discusiones hechas por Lucas (1970) y Romer (1987), aclaran que este es un resultado usual, y que se puede identificar principalmente en el caso del capital. Este resultado de todas formas puede ser señal de que los datos no son sufiientes o simplemente es la respuesta al valor medio de toda la serie utilizada, pues en últimas las elasticidades de esta función trascendental es variable.

Teniendo en cuenta lo anterior, para analizar los datos es necesario tomar sus valores absolutos. De esta forma, como se pudo observar, tan sólo el trabajo es un factor cuyo valor absoluto es elástico. Esto significa que la producción de compost es bastante sensible a la variación de dicho factor.

Por su parte, descomponer este resultado de forma analítica resulta necesario, pues la producción de compost no es precisamente muy intensiva en mano de obra (como se muestra en el estudio realizado por Park et al. (2002), el compostaje puede ser producido incluso desde casa), por lo tanto, los efectos de la misma no deberían ser notorios.

No obstante, para ilustrar este escenario, es necesario imaginar una situación en la que el capital, es decir las plantas de tratamiento, permanecen relativamente constantes en el tiempo. Esta capacidad instalada puede permanecer así durante mucho tiempo, por lo tanto un cambio marginal en este factor es poco probable acorde a los datos disponibles, mientras que el número de empleados dedicados a la disposición de residuos y tratamiento de aguas sí puede variar considerablemente durante el mismo periodo, pues es una fuente de empleo importante independientemente el nivel de desarrollo (Organización de las Naciones Unidas, 2017), afectando la producción de compostaje de forma sustancial, ya que dada la necesidad de pocos trabajadores para este efecto específico, un cambio unitario o marginal en el nivel de empleo influiría directamente sobre la producción.

Explicado de otra forma, lo que se pretende decir es que para producir compost se requieren pocos empleados a cargo de varios implementos; el incremento marginal de cualquier implemento, digamos por ejemplo, las camas de compostaje, no influye en la producción de compost como influiría la contratación de una persona más, pues usualmente un solo individuo puede verificar el estado de varias camas de producción de compost, así que la instalación de una nueva cama (o una nueva planta de tratamiento) para producir compost, no generaría, marginalmente hablando, la misma cantidad de producción que si puede generar un empleado más.

Sobre la base de lo anterior, empiezan a aparecer otros conceptos que también se encuentran en los resultados. Las elasticidades de sustitución de Allen (Tabla 6), establecen que el capital es complementario con los demás factores. Este punto se puede explicar siguiendo el mismo ejemplo anterior. Se puede suponer la necesidad de construir una nueva planta de tratamiento, esta planta por sí sola no implica un aumento considerable en la producción de compost suponiendo que la demanda y otras variables del mercado permanecen constantes. Sin embargo, esta planta requiere que los residuos sean dispuestos allí y una vez tratados, deben ser llevados a los puntos rurales donde puedan ser usados para el proceso anaeróbico de compostaje, en otras palabras, el capital se relaciona directamente con el trabajo y los insumos, de esta forma el capital complementa a los otros dos factores.

Caso contrario ocurre con el trabajo y los residuos sólidos. Un aumento en la generación de residuos no viene acompañada necesariamente por un aumento de la mano 
de obra y viceversa, ni mucho menos de la producción ${ }^{6}$. La generación de residuos no garantiza por si sola que estos vayan a ser usados en la producción de compost; y si este fuera el caso, la mano de obra existente relacionada directamente podría permanecer constante, incluso si fuera necesario la instalación de más plantas de tratamiento.

Lo anterior va en concordancia en los sistemas y métodos de compostaje del trabajo de Misra \& Hiraoka (2003) en donde se exponen las características de cada metodología, y se destaca que en los países en donde hay posibilidad de utilizar maquinaria para el proceso, el trabajo es utilizado en menor medida e intensidad.

Para terminar, el punto anterior lleva directamente a otro concepto tratado durante la presentación de los resultados, este es la separabilidad. Acorde a los cálculos, el trabajo agrícola en conjunto con las plantas instaladas son separables de los residuos sólidos. Dado que exista un aumento (o disminución) en la cantidad de residuos sólidos generados por la municipalidad, pero las plantas de tratamiento y el número empleados permanezcan constantes, estos dos últimos factores van a aumentar (o disminuir) su producto marginal, en la misma proporción del aumento (o disminución) de los residuos. De esta manera, el capital y el trabajo responden conjuntamente a los cambios que se produzcan en la generación de residuos municipales, pero este proceso no pasa en el sentido contrario, expresado de otra forma, significa que, si existen más residuos, puede impulsarse la producción marginal de compost.

\section{Conclusiones}

El propósito de este trabajo fue acercarse a una función de producción de compost para diferentes países. Luego de aplicar las respectivas pruebas, se encontró que la mejor forma funcional fue la translogarítmica. Con esta función las elasticidades de los factores son variables en el tiempo y para este caso entre individuos.

Dentro de los resultados para destacar se encontró que ninguna de las elasticidades calculadas en sus puntos medios cumplió con los signos esperados. Sin embargo, este tipo de resultados ya se había evidenciado en otros trabajos realizados previamente.

Dada la relativa escasez en el uso del trabajo para la producción de compostaje y la relativa estacionariedad de los factores capital e insumos. El primer factor que se mencionó es bastante elástico, mientras que los otros dos factores no generan una sensibilidad considerable en la fabricación de compost.

Además, se calcularon las elasticidades de cada uno de los países objeto de estudio. El principal hallazgo es que, entre algunos de ellos, los cambios son sustanciales y muy alejados del promedio. En ciertos países los signos y las predicciones teóricas parecen cumplirse para la producción de compost.

Sumado a lo anterior, también se calcularon las elasticidades de sustitución de Allen. Con estas pruebas se pudo verificar que el capital, representado por el índice de instalación de nuevas plantas de tratamiento, es complementario con los demás factores de producción. Caso contrario ocurre con el trabajo y los residuos municipales generados, es decir los insumos, estos factores resultan sustitutivos para todo el grupo de países.

Finalmente, se realizaron algunas pruebas de separabilidad de los factores, llegando a la conclusión que el capital y el trabajo, son separables de los insumos en esta función de producción.

\section{Agradecimientos}

Proyecto de investigación INV-ECO-2333 "Análisis económico del proceso de vermicompostaje como alternativa de disposición eficiente para biosólidos PTAR UMNG (Cajicá)." financiado por la Vicerrectoría de Investigaciones de la Universidad Militar Nueva Granada en la vigencia 2017.

\footnotetext{
${ }^{6}$ Esto explica la inelasticidad del factor. El lector puede notar que, dado que es el caso opuesto al trabajo que es escaso, este factor no es elástico. Un incremento en la generación de residuos puede permanecer en el tiempo hasta que sea necesario la instalación de una nueva planta o hasta que exista un cambio tecnológico, esto significa que su cambio no va impactar la producción de compost, pues en últimas es un factor relativamente barato de obtener.
} 


\section{Referencias}

Allen, R. G. D. (1938). Mathematical analysis for economists. London: MacMillan.

Arancon, N., Edwards, C., Bierman, P., Welch, C., \& Metzger, J. (2004). Influences of vermicomposts on field strawberries: 1. effects on growth and yields. Bioresource Technology, 93(2), 145-153.

Berndt, E., \& Christensen, L. (1973). The translog function and the substitution of equipment, structures, and labor in U.S. manufacturing 1929-1968. Journal of Econometrics, 1(1), 81-114.

Camargo, D., Cardona, O., \& González, J. (2017a). Valuación económica del vermicompostaje, ¿Vacíos en la literatura? In VII Encuentro Nacional III Internacional de Ciencia y Tecnología. Bogotá D.C.: Escuela Militar de Suboficiales, Unidad de Investigación.

Camargo, D., Cardona, O., \& González, J. (2017b). Una aproximación a la función de producción de compost para 33 países. In III Reunión Iberoamericana de Socioeconomía. Bolivar, Colômbia: Universidad Tecnológica de Bolivar.

Christensen, L., Jorgenson, D., \& Lau, L. (1973). Transcendenta logarithmic production frontiers. Revista de Economia, 55(1).

Felipe, J., \& Adams, F. G. (2005). A theory of production" the estimation of the Cobb-Douglas function: a retrospective view. Eastern Economic Journal, 31(3), 427-445.

Folefack, A. J. (2009). The substitution of mineral fertilizers by compost from household waste in Cameroon: economic analysis with a partial equilibrium model. Waste Management \& Research, 27(3), 207-223.

Folefack, A. J., \& Adamowski, J. F. (2012). Application of the Von Thünen model in determining optimal locations to transport compost for crop production outside of Yaoundé, Cameroon. Journal of Human Ecology, 39(2), 125-143.

Grabowski, R. (1985). Technical efficiency in Nepalese agriculture. Journal of Developing Areas, 19(4), $515-$ 526.

Greene, W. (1998). Análisis econométrico. Madrid: Prentice Hall.

Griliches, Z., \& Mairesse, J. (1995). Production functions: the search for identification. Cambridge: National Bureau of Economic Research.

Ibarra Salazar, J., \& García Pérez, F. (2013). Elasticidades de sustitución y separabilidad de los factores productivos de la industria maquiladora. Estudios Fronterizos, 14(28), 155-185.

Jackson, L., Ramirez, I., Yokota, R., Fennimore, S., Koike, S., Henderson, D., Chaney, W. E., Calderón, F. J., \& Klonsky, K. (2004). On-farm Assessment of Organic Mmatter and Ttillage Management on Vegetable Yield, Soil, Weeds, Pests, and Economics in California. Agriculture, Ecosystems \& Environment, 103(3), 443-463.

Klette, T. J., \& Griliches, Z. (1996). The inconsistency of common scale estimators when output prices. Journal of Applied Econometrics, 11(4), 343-361.

Lucas, R. (1970). Capacity, overtime, and empirical production functions. The American Economic Review, 60(2), 23-27.

Misra, R. V., \& Hiraoka, H. (2003). On-farm composting methods. Rome: UN-FAO.

Organización de las Naciones Unidas. (2017). Aguas residuales el recurso desaprovechado. París: UNESCO-ONU Agua.

Park, W. M., Lamons, K. S., \& Roberts, R. K. (2002). Factors associated with backyard composting behavior at the household level. Agricultural and Resource Economics Review, 31(2), 147-156.

Ramíres, A. (2015). Ajuste de una función de producción al sector financiero en Colombia. Revista Facultad de Ciencias Económicas: Investigación y Reflexión, 23(1).

Romer, P. M. (1987). Crazy explanations for the productivity slowdown. NBER Macroeconomics Annual, 2 , 163-202.

Terrones-Cordero, A., \& Sánchez-Torres, Y. (2010). Demandas de insumos de la producción agrícola en México, 1975-2011. Universidad y Ciencia, 26(1), 82-91.

Submetido: 8/Mar./2018.

Aceito: 27/Out./2019

Códigos JEL: D24, Q15, Q56. 
Anexo A1 Salidas en Stata 12.1 del modelo sin efectos

\begin{tabular}{|c|c|c|c|c|c|c|}
\hline & SS & Df & MS & & $\mathbf{N}$ & 371 \\
\hline & & & & & $F(9,361)$ & 163.18 \\
\hline Modelo & 1317.823 & 9 & 146.425 & & Prob $>\mathrm{F}$ & 0.000 \\
\hline Residual & 323.938 & 361 & 0.897 & & R-cuadrado & 0.8027 \\
\hline & & & & & Adj R-cuadrado & 0.7978 \\
\hline Total & 1641.76102 & 370 & 4.43719194 & & Raíz MSE & 0.94728 \\
\hline LnY & Coef. & Std. Err. & $\mathrm{T}$ & $P>t$ & \multicolumn{2}{|c|}{ Intervalo de confianza 95\% } \\
\hline LnK & -6.977 & 5.337 & -1.31 & 0.192 & -17.472 & 3.518 \\
\hline LnL & -3.026 & 1.331 & -2.27 & 0.024 & -5.644 & -0.408 \\
\hline LnM & -0.001 & 0.905 & 0.00 & 0.999 & -1.780 & 1.779 \\
\hline $\operatorname{Ln} 2 \mathrm{~K}$ & 0.896 & 1.090 & 0.82 & 0.412 & -1.248 & 3.041 \\
\hline $\operatorname{Ln} 2 \mathrm{~L}$ & -0.659 & 0.140 & -4.72 & 0.000 & -0.934 & -0.384 \\
\hline $\operatorname{Ln} 2 \mathrm{M}$ & 0.029 & 0.040 & 0.73 & 0.463 & -0.049 & 0.107 \\
\hline LnKLnL & 0.552 & 0.269 & 2.05 & 0.041 & 0.024 & 1.081 \\
\hline LnKLnM & 0.186 & 0.170 & 1.1 & 0.273 & -0.148 & 0.520 \\
\hline LnLLnM & 0.082 & 0.046 & 1.8 & 0.073 & -0.008 & 0.172 \\
\hline _cons & 20.668 & 13.912 & 1.49 & 0.138 & -6.690 & 48.027 \\
\hline
\end{tabular}

Anexo A2 Salidas en Stata 12.1 del modelo con efectos fijos

\begin{tabular}{|c|c|c|c|c|c|c|}
\hline \multicolumn{5}{|c|}{ Fixed-effects (within) regression } & $\mathbf{N}$ & 371 \\
\hline \multirow{2}{*}{\multicolumn{5}{|c|}{ Group variable: unit_id }} & Grupos & 31 \\
\hline & & & & & \multicolumn{2}{|c|}{ Obs por grupo } \\
\hline \multicolumn{3}{|c|}{ R-sq: within $=0.1706$} & & & minimo & 5 \\
\hline \multicolumn{3}{|c|}{ between $=0.0014$} & & & promedio & 12 \\
\hline \multirow{2}{*}{\multicolumn{3}{|c|}{ overall $=0.0045$}} & & & maximo & 13 \\
\hline & & & & & $F(9,331)$ & 7.56 \\
\hline \multicolumn{5}{|c|}{$\operatorname{corr}\left(u_{-} i, X b\right)=-0.5894$} & Prob $>\mathrm{F}$ & 0 \\
\hline LnY & Coef. & Std. Err. & $\mathrm{T}$ & $\mathrm{P}>\mathrm{t}$ & \multicolumn{2}{|c|}{ Intervalo de confianza 95\% } \\
\hline LnK & 15.322 & 5.216 & 2.94 & 0.004 & 5.061 & 25.583 \\
\hline LnL & 5.916 & 2.028 & 2.92 & 0.004 & 1.927 & 9.905 \\
\hline LnM & -1.130 & 2.074 & -0.54 & 0.586 & -5.210 & 2.950 \\
\hline $\operatorname{Ln} 2 \mathrm{~K}$ & -2.316 & 1.015 & -2.28 & 0.023 & -4.312 & -0.320 \\
\hline $\operatorname{Ln} 2 \mathrm{~L}$ & -1.324 & 0.317 & -4.17 & 0.000 & -1.948 & -0.700 \\
\hline
\end{tabular}




\begin{tabular}{ccccccc}
\multicolumn{2}{c}{ Fixed-effects (within) regression } & & & $\mathbf{N}$ & 371 \\
Ln2M & 0.417 & 0.248 & 1.68 & 0.093 & -0.070 & 0.905 \\
LnKLnL & -0.753 & 0.330 & -2.28 & 0.023 & -1.402 & -0.104 \\
LnKLnM & -0.497 & 0.185 & -2.68 & 0.008 & -0.862 & -0.133 \\
LnLLnM & -0.207 & 0.174 & -1.19 & 0.234 & -0.549 & 0.135 \\
_cons & -26.101 & 16.620 & -1.57 & 0.117 & -58.795 & 6.592 \\
sigma_u & 2.7293523 & & & & & \\
sigma_e & 0.63125364 & & & & & \\
rho & 0.94922413 & & & & & \\
\hline
\end{tabular}

Anexo A3 Salidas en Stata 12.1 del modelo con efectos aleatorios

\begin{tabular}{|c|c|c|c|c|c|c|}
\hline \multicolumn{3}{|c|}{ Random-effects GLS regression } & & & $\mathbf{N}$ & 371 \\
\hline \multirow{2}{*}{\multicolumn{2}{|c|}{ Group variable: unit_id }} & & & & Grupos & 31 \\
\hline & & & & & \multicolumn{2}{|c|}{ Obs por grupo } \\
\hline \multicolumn{2}{|c|}{ R-sq: within $=0.0597$} & & & & minimo & 5 \\
\hline \multicolumn{2}{|c|}{ between $=0.8298$} & & & & promedio & 12 \\
\hline \multicolumn{2}{|c|}{ overall $=0.7572$} & & & & maximo & 13 \\
\hline & & & & & Wald chi2(9) & 211.22 \\
\hline \multicolumn{3}{|c|}{$\operatorname{corr}\left(u_{-} i, X\right)=0$ (assumed) } & & & Prob > chi2 & 0 \\
\hline $\operatorname{LnY}$ & Coef. & Std. Err. & Z & $\mathrm{P}>\mathrm{Z}$ & [95\% Conf. & Interval] \\
\hline LnK & 14.794 & 5.237 & 2.83 & 0.005 & 4.530 & 25.057 \\
\hline LnL & 3.267 & 1.659 & 1.97 & 0.049 & 0.016 & 6.518 \\
\hline LnM & 1.506 & 1.167 & 1.29 & 0.197 & -0.782 & 3.794 \\
\hline Ln2K & -2.502 & 1.036 & -2.41 & 0.016 & -4.533 & -0.470 \\
\hline $\operatorname{Ln} 2 \mathrm{~L}$ & -0.909 & 0.251 & -3.63 & 0.000 & -1.400 & -0.418 \\
\hline $\operatorname{Ln} 2 \mathrm{M}$ & 0.170 & 0.091 & 1.86 & 0.062 & -0.009 & 0.349 \\
\hline LnKLnL & -0.499 & 0.293 & -1.7 & 0.088 & -1.073 & 0.075 \\
\hline LnKLnM & -0.382 & 0.168 & -2.28 & 0.023 & -0.710 & -0.054 \\
\hline LnLLnM & -0.055 & 0.097 & -0.57 & 0.567 & -0.245 & 0.134 \\
\hline _cons & -39.461 & 14.462 & -2.73 & 0.006 & -67.805 & -11.117 \\
\hline sigma_u & 0.6635301 & & & & & \\
\hline sigma_e & 0.63125364 & & & & & \\
\hline rho & 0.52491259 & & & & & \\
\hline
\end{tabular}


Anexo A4 Salidas en Stata 12.1 del contraste de Hausman

\begin{tabular}{|c|c|c|c|}
\hline \multicolumn{3}{|c|}{ Coefficients } & \multirow{2}{*}{$\operatorname{sqrt}\left(\operatorname{diag}\left(V_{-}\right.\right.$b-V_B $)$) } \\
\hline (b) & (B) & (b-B) & \\
\hline sefe & Refe & Difference & S.E. \\
\hline
\end{tabular}

$\begin{array}{ccccc}\text { LnK } & 15.322 & 14.794 & 0.529 & \cdot \\ \text { LnL } & 5.916 & 3.267 & 2.649 & 1.166 \\ \text { LnM } & -1.130 & 1.506 & -2.636 & 1.714 \\ \text { Ln2K } & -2.316 & -2.502 & 0.185 & \cdot \\ \text { Ln2L } & -1.324 & -0.909 & -0.415 & 0.195 \\ \text { Ln2M } & 0.417 & 0.170 & 0.247 & 0.230 \\ \text { LnKLnL } & -0.753 & -0.499 & -0.254 & 0.152 \\ \text { LnKLnM } & -0.497 & -0.382 & -0.115 & 0.079 \\ \text { LnLLnM } & -0.207 & -0.055 & -0.152 & 0.145\end{array}$

Test: Ho: $\quad$ difference in coefficients not systematic

chi2 $(9)=(b-B)^{\prime}\left[\left(V_{-} b-V_{-} B\right)^{\wedge}(-1)\right](b-B)$

52.85

Prob $>$ chi $2=0.0000$

( $V_{-}$b-V_B is not positive definite)

Anexo A5 Salidas en Stata 12.1 del constraste de Breusch-Pagan

Breusch and Pagan Lagrangian multiplier test for random effects

$\operatorname{LnY}\left[\right.$ unit_id,t] $=X b+u[$ unit_id $]+e\left[u n i t \_i d, t\right]$

Estimated results:

$\begin{array}{lll} & \text { Var } & \text { sd=sqrt(Var) } \\ \text { LnY } & 4.437192 & 2.106464 \\ \text { E } & 0.3984812 & 0.6312536 \\ \text { U } & 0.4402722 & 0.6635301\end{array}$

Test: $\quad \operatorname{Var}(\mathrm{u})=0$

$\operatorname{chibar2}(01)=341.13$

Prob $>$ chibar $2=0.000$ 
Estimación de una función para la producción de compost: el caso europeo

Anexo A6 Salidas en Stata 12.1 de la prueba F para el modelo translogaritmico vs Cobb-Douglas

- test $\operatorname{Ln} 2 \mathrm{~K}=\mathrm{Ln} 2 \mathrm{~L}=\operatorname{Ln} 2 \mathrm{M}=\operatorname{Ln} K \operatorname{Ln} L=\operatorname{Ln} K \operatorname{Ln} M=\operatorname{Ln} \operatorname{LLn} M=0$
( 1) $\operatorname{Ln} 2 \mathrm{~K}-\operatorname{Ln} 2 \mathrm{~L}=0$
( 2) $\operatorname{Ln} 2 \mathrm{~K}-\operatorname{Ln} 2 \mathrm{M}=0$
( 3$) \quad \operatorname{Ln} 2 K-\operatorname{LnKLnL}=0$
( 4) $\operatorname{Ln} 2 \mathrm{~K}-\operatorname{LnKLnM}=0$
( 5) Ln2K - LnLLnM = 0
( 6) $\quad \operatorname{Ln} 2 \mathrm{~K}=0$

$\begin{aligned} \text { F }(3,331) & =5.56 \\ \text { Prob }>F & =0.0000\end{aligned}$

